Z. Klin. Chem. Klin. Biochem.

12. Jg. 1974 , S. $313-320$

\title{
Über die Einwirkung von Pyridinolcarbamat auf die Bildung saurer Glykosaminoglykane und Kol- lagen im Bindegewebe der experimentellen Lebercirrhose der Ratte
}

\author{
Von $A$. Delbrück \\ Institut für Klinische Chemie, Abt. II der Medizinischen Hochschule Hannover, Zentrallaboratorium am Kranken- \\ haus Oststadt
}

(Eingegangen am 7. Januar/4. Februar 1974)

\section{Herrn Prof. Dr. Dr. h.c. Theodor Bücher zum 60. Geburtstag}

\begin{abstract}
Am Modell der durch Thioacetamid induzierten Lebercirrhose der Ratte wurde der Einfluß von Pyridinolcarbamat auf die Synthese und intrazelluläre Deponierung von Grundsubstanzanteilen und Faserproteinen untersucht. Hierbei ergab sich, daß der Gehalt an sauren Glykosaminoglykanen sowie Kollagen in der mit Pyridinolcarbamat behandelten Versuchsgruppe signifikant gegenüber der Vergleichsgruppe herabgesetzt war. Qualitative Verschiebungen im Verteilungsmuster der sauren Glykosaminoglykane konnten nicht beobachtet werden. Die morphologischen Befunde entsprachen den Ergebnissen der biochemischen Analysen. Es wird eine Hemmung der Synthese der Interzellularsubstanzen als Ursache der antiproliferativen Wirkung von Pyridinolcarbamat diskutiert.
\end{abstract}

The effect of pyridinolcarbamate on the synthesis of glycosaminoglycans and collagen in connective tissue in experimental induced liver cirrhosis in rats

Experimental liver cirrhosis was induced in rats by the application of thioacetamide. Simultaneously fed pyridinolcarbamate resulted in a significantly lower level of glycosaminoglycans as well as collagen, as compared with the pyridinolcarbamate untreated animals. No qualitative changes in the glycosaminoglycan distribution pattern were observed. The biochemical findings corresponded to the morphological state of the liver tissue. The antiproliferative effect of pyridinolcarbamate is thought to be caused by an inhibition of the synthesis of intercellular substances.

Pyridinolcarbamat (2,6- Bis (hydroxymethyl)pyridin- bis -N-methylcarbamat) ist eine stabile, schwer lösliche, weiße, kristalline Substanz, über deren antiatherogene Wirksamkeit erstmals von Shimamoto et al 1965 berichtet wurde (1). In der Folgezeit wurde sowohl experimentell wie klinisch der Frage nach dem Wirkungsmechanismus dieses Stoffes in der Prävention der Arteriosklerose nachgegangen $(2,3)$. Unter anderem ist die Wirkung von Pyridinolcarbamat auch am Modell des Osteolathyrișus untersucht worden. Yamamura (4) demonstrierte morphologisch einen präventiven Effekt von Pyridinolcarbamat auf die Ausbildung der pathologischen Veränderungen am Gefäßbindegewebe und am Knochen bei experimentell durch Applikation von $\beta$-Aminoproprionitril herbeigeführtem Lathyrismus der Ratte. Er diskutierte eine Hemmung der beim Osteolathyrismus erhöhten Prokollagen-Synthese durch Pyridinolcarbamat. Dieser Befund legt die Vermutung nahe, daß die Wirksamkeit des Pyridinolcarbamates bei der Verhütung von degenerativen Gefäßerkrankungen nicht so sehr auf dem AntiBradykinin-Effekt oder auf einer Beeinflussung des Blutgerinnungssystemes beruht, als vielmehr auf einer direkten Einflußnahme auf den Stoffwechsel des Gefäßbindegewebes. Unter dieser Hypothese schien es angezeigt, die Wirksamkeit von Pyridinolcarbamat an einem Modell gestörter Bindegewebsbildung zu untersuchen, das dem Ein- fluß der obengenannten Faktoren weitgehend entzogen ist. Hierzu bot sich das Modell der experimentell durch die Gabe von Thioacetamid erzeugten Lebercirrhose der Ratte an (5), bei dem infolge eines vorangehenden Parenchymzellschadens eine mesenchymale Reaktion ausgelöst wird, die in eine unkontrollierte pathologische Narbenbildung einmündet. Als biochemische Parameter zur quantitativen Bestimmung der Vermehrung bindegewebiger Struktursubstanzen wurden die Gewebsspiegel der sauren Glykosaminoglykane und der Faserproteine (Kollagenfraktionen) herangezogen, um auf die vorgelegte Frage nach einer Wirksamkeit des Pyridinolcarbamates auf den Bindegewebsstoffwechsel Antwort zu geben und zu prüfen, ob gegebenenfalls aus unterschiedlichem Verhalten der Spiegel der sauren Glykosaminoglykane und der Faserproteine eine nähere Differenzierung der Wirkungsweise von Pyridinolcarbamat möglich ist.

\section{Material und Methoden}

\section{Tiermaterial und Haltung}

59 o Wistar-Ratten $\left.{ }^{1}\right)$, 180g Anfangsgewicht, Einzelkäfige, Standardpreßfutter Altromin Rogge, Flüssigkeitsaufnahme ad libitum. Wöchentliche Gewichtskontrolle und gravimetrische Bestimmung des Flüssigkeitsverbrauches.

1) Zentralinstitut für Versuchstierforschung, Hannover 
Tab. 1. Analysengang - Übcrsicht

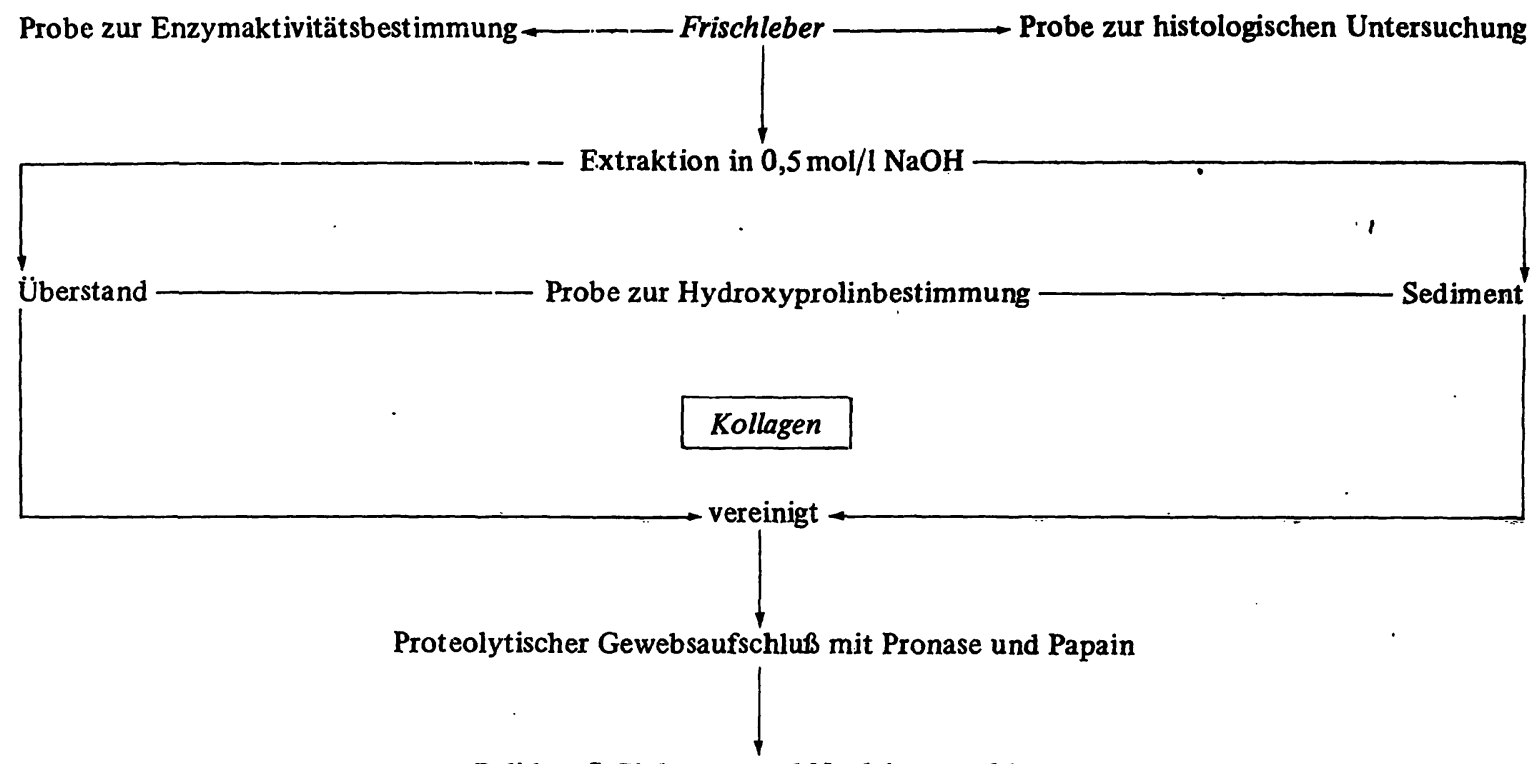

Polidase-S Glykogen- und Nucleinsäureabbau

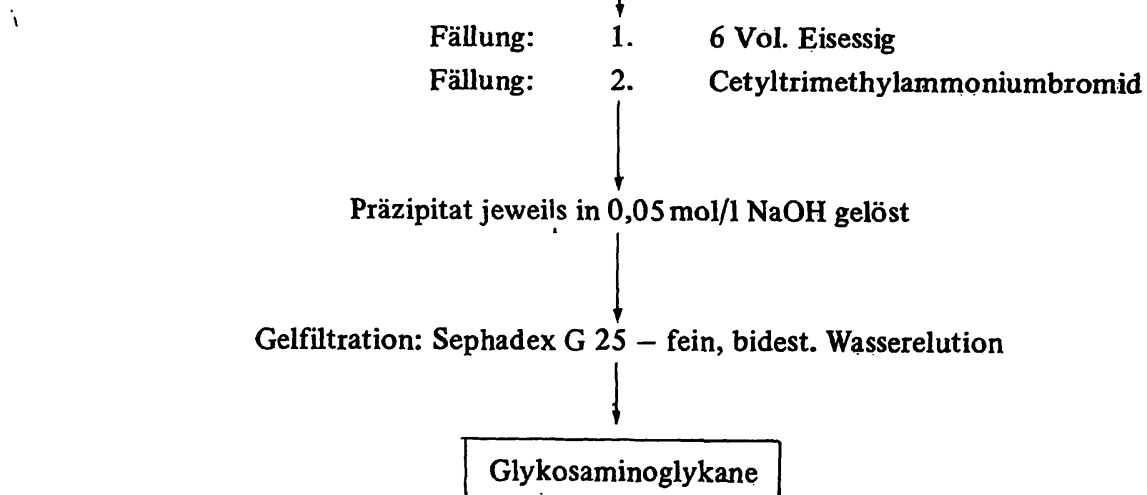

Glucuronsäurebestimmung nach Dische

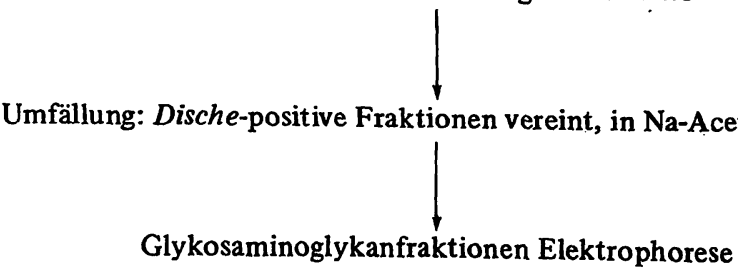

Versuchsanordnung

Vergleichsgruppe (I, Thioacetamid)

Applikation subcutan $5 \mathrm{mal}$ wöchentlich, 1.-12. Woche $160 \mathrm{mg} /$ $\mathrm{kg}$ Körpergewicht/Woche, 13.-22. Woche $280 \mathrm{mg} / \mathrm{kg}$ Körpergewicht/Woche als 1 prozentige Thioacetamidlösung. Injektionsstelle in der Nackenregion.

Versuchsgruppe (II, Thioacetamid und Pyridinolcarbamat) Applikation von Thioacetamid subcutan wie Gruppe I, zusätzlich in Trinkwasser 0,2\% Pyridinolcarbamat, Aufnahme ad libitum. Durchschnittliche Pyridinolcarbamat-Aufnahme während der Versuchsdauer: $960 \mathrm{mg} / \mathrm{kg}$ Körpergewicht/Woche.

\section{Kontrollgruppe (III, Normaltiere)}

Standardfutter und Trinkwasser ad libitum.

\section{Versuchsdauer}

5 Monate. Kontrolle der Cirrhose-Entwicklung nach 12 Wochen (2 Tiere der Gruppe I), nach 16 Wochen (1 Tier der Gruppe I). Bei Versuchsende Opferung der Tiere durch Dekapitation über 2 Wochen verteilt, zu jedem Termin gleiche.Tierzahl aus Gruppe
I und II. Auffangen des Blutes, Präparation und makroskopischmorphologische Klassifikation der Lebern entsprechend der Oberflächenbeschaffenheit, der Konsistenz und des Ausmaßes der Lappung in die Klassen 0 (unverändert) bus 6 (ausgeprägte Cirrhose). Probeentnahme zur mikroskopischen Untersuchung ${ }^{2}$ ), jeweils am vorderen Rand des rechten Leberlappens. Beurteilung der mikroskopischen Veränderungen a nalog zur makroskopischen Klassifikation in die Gruppen 0 bis 6 . Weitere Probeaufbereitung s. Tabelle 1.

Bestimmung des Gewebsgehaltes an Kollagen (alkalilösliche und alkaliunlösliche Fraktion)

Extraktion der hydroxyprolinhaltigen Proteine bzw. Peptide aus Acetontrockenpulver $(1 \mathrm{~g} / 30 \mathrm{ml}$ 0,5 mol/1 $\mathrm{NaOH})$ unter Rühren über $20 \mathrm{~h}$ bei $+4^{\circ} \mathrm{C}$. Abtrennen des Unlöslichen durch Zentrifugation über $30 \mathrm{~min}$ bei $19500 \mathrm{~g}$ (MSE Superspeed 50) (6).

2) Der Autor dankt den Herren Prof. Dr. Mohr und Dr. Hilfrich, Abt: fuir experimentelle Pathologie, Institut für Pathologie der Med. Hochschule Hannover, für die Ausführung der histologischen Untersuchungen des Lebermaterials. 
Hydroxyprolinbestimmung: Salzsaure Hydrolyse in $6 \mathrm{~mol} / 1 \mathrm{HCl}$ über $24 \mathrm{~h}$ bei $110^{\circ} \mathrm{C}$. Abdampfen der $\mathrm{HCl}$ im Stickstoffstrom bei $56^{\circ} \mathrm{C}$, lösen des Rückstandes in $\mathrm{H}_{2} \mathrm{O}$, Filtration und photometrische Bestimmung am Filtrat nach Stegemann (7), Hydrolyse am Unlöslichen nach Trocknen des Sedimentes im Vakuum und Einwaage der Probe.

Bestimmung der Gewebsspiegel saurer Glykosam inoglykane modifiziert nach (8)

Gewebsproteolyse am vereinigten und alkalilöslichen und -unlöslichen Gewebsanteil der Leber mit Pronase ${ }^{3}$ ) und Papain. Glykogen- und Nucleinsäureabbau durch Polidase- ${ }^{4}$ ). Ausfällung der Rohfraktion mit 6-fachem Volumen Eisessig. Wiederaufnahme des Sedimentes in $0,05 \mathrm{~mol} / 1 \mathrm{NaOH}$ und Ausfällung der sauren Glykosaminoglykane mit Cetyltrimethylammoniumbromid ${ }^{5}$ ). Abtrennen der sauren Glykosaminoglykane aus dem Cetyltrimethylammoniumbromid-Glykosaminoglykan-Komplex durch $\mathrm{NaCl}$-gesättigten Äthanol. Lösen des Präzipitates in $0,05 \mathrm{~mol} / 1$ $\mathrm{NaOH}$ und Abtrennen von Begleitstoffen über Sephadex-G-25fein $^{6}$ ) (Säulenbett $1,2 \times 140 \mathrm{~cm}$ ). Elution mit aqua dem. und fraktionierter Kollektion des Eluates. Quantitative Glucuronsäurebestimmung nach Dische (9). Der Gehalt an sauren Glykosaminoglykanen des Gewebes wurde in mg Glucuronsäure/g Acetontrockenpulver ohne Berïcksichtigung der Abweichung des spezifischen Extinktionskoeffizienten der einzelnen Fraktionen der sauren Glykosaminoglykane vom Extinktionskoeffizienten des Glucuronsäurestandards ausgedrückt.

Enzymaktivitätsbestimmungen

Glutamat-Pyruvat-Transaminase (EC 2.6.1.2) und Glutamat-Oxatacetat-Transaminase (EC 2.6.1.1) nach Schmidt \& Schmidt (10). - Glutamatdehydrogenase (EC 1.4.1.3): Triäthanolamin-HCl-Puffer $50 \mathrm{mmol} / \mathrm{l}$, pH 8,0 . NADH $0,1 \mathrm{mmol} / \mathrm{l}$, Ammoniumacetat $100 \mathrm{mmol} / 1$, Äthylendiamintetratacetat $2,5 \mathrm{mmol} / 1,1 \mathrm{mmol} / 1$ 2-Oxoglutarat $9 \mathrm{mmol} / 1.25^{\circ} \mathrm{C}$. Alkalische Phosphatase (EC 3.1.3.1): nach Hausamen et al, Kollagenpeptidase: nach Wünsch und Heidrich sowie Strauch und Vencelj $(12,13,14)$.

Nachweis von Pyridinolcarbamat und Thioacetamid im Lebergewebe dünnschichtchromatographisch modifiziert nach (15).

Bestimmung der Konzentration von Bilirubin im Serum nach Jendrassik und Grof (16).

Bestimmung von Cholesterin im Serum nach Watson (17).

\section{Ergebnisse}

Die Ergebnisse der Untersuchungen sind in den Tabellen 2 und 3 bzw. Abbildungen 1-5 zusammengefaßt dargestellt. Bei subcutaner Applikation des Thioacetamids in den angegebenen Dosen kam es innerhalb des Versuchszeitraumes von 21 Wochen in der mit Pyridinolcarbamat unbehandelten Vergleichsgruppen (1) bei 19 von 22 Lebern zum Vollbild der Cirrhose, in den restlichen 3 Fällen waren schwere morphologische Veränderungen nachweisbar. Demgegenüber ließ die makroskopische Beurteilung der mit Pyridinolcarbamat zusätzlich behandelten Vergleichsgruppe (II) nur in einzelnen Fällen Veränderungen im Sinne einer Cirrhose erkennen. Die Mikroskopische Untersuchung der Versuchsgruppe (II) zeigte jedoch in der Hälfte der Fälle fortgeschrittene Stadien der Lebercirrhose (Abb. 1, Abb. 4). Den ausgeprägten Veränderungen im morphologischen Aufbau der Leber entsprachen die Veränderungen der Gewebsspiegel der sauren Glykosaminoglykane (Tab. 2, Abb. 2). Bei der

\footnotetext{
3) Serva GmbH, Heidelberg

4) Schwarz Laboratories, New York/USA

5) Fluka AG, Buchs, Schweiz

6) Pharmacia, Upsala, Schweden
}
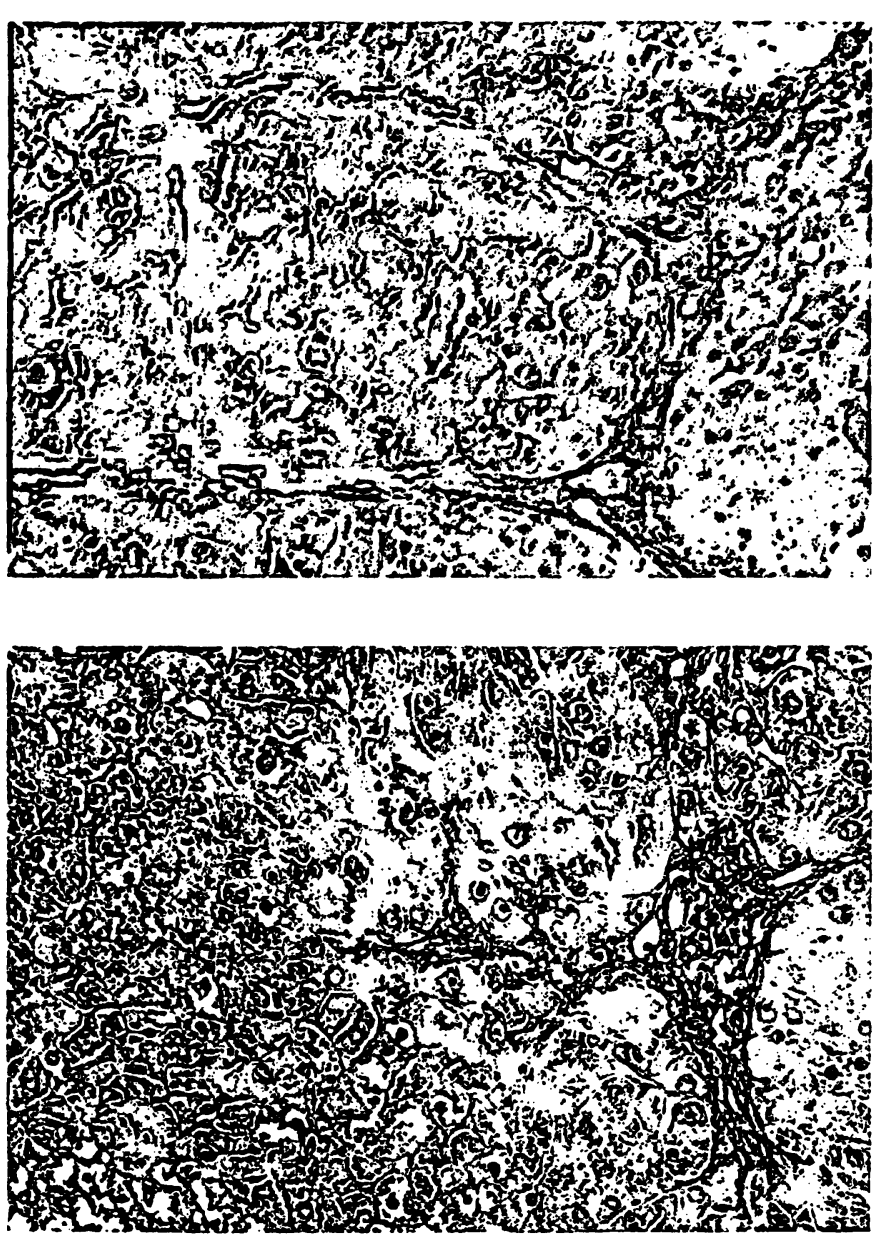

Abb. 1. Experimentelle Lebercirrhose der Ratte. Histologisches Bild (van Gieson, Vergrößerung 225-fach),

a) bei alleiniger Gabe von Thioacetamid (Gruppe I),

b) bei gleichzeitiger Verabfolgung von Thioacetamid und Pyridinolcarbamat (Gruppe II), jeweils nach 5 Monaten Versuchsdauer.

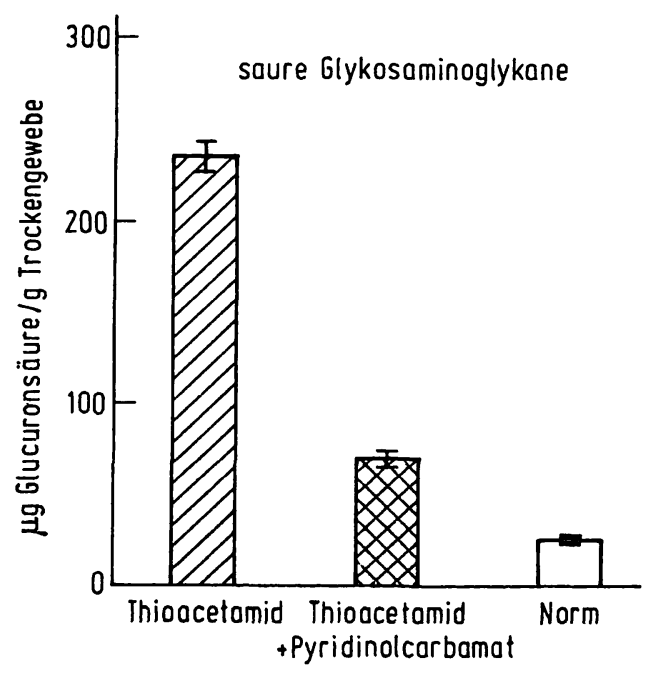

Abb. 2. Gewebsspiegel saurer Glykosaminoglykane, ausgedrückt als Glucuronsäuregehalt in den Versuchsgruppen I (Thioacetamid) und II (Thioacetamid + Pyridinolcarbamat) und in unbehandelten Kontrollticren. I = Standardabweichung der Mittelwerte 
Tab. 2. Biochemische Parameter der Bindegewebsbildung.

Mittelwert, Standardabweichung, Minimum- und Maximumwerte, Median, Standardabweichung der Mittelwerte $\left(s_{\overline{\mathrm{x}}}\right)$ und Signifikanz der Gruppenunterschiede nach dem $t$-Test für unverbundene Stichproben.

ATG Acetontrockenpulver

TAA Thioacetamid

PC Pyridinolcarbamat

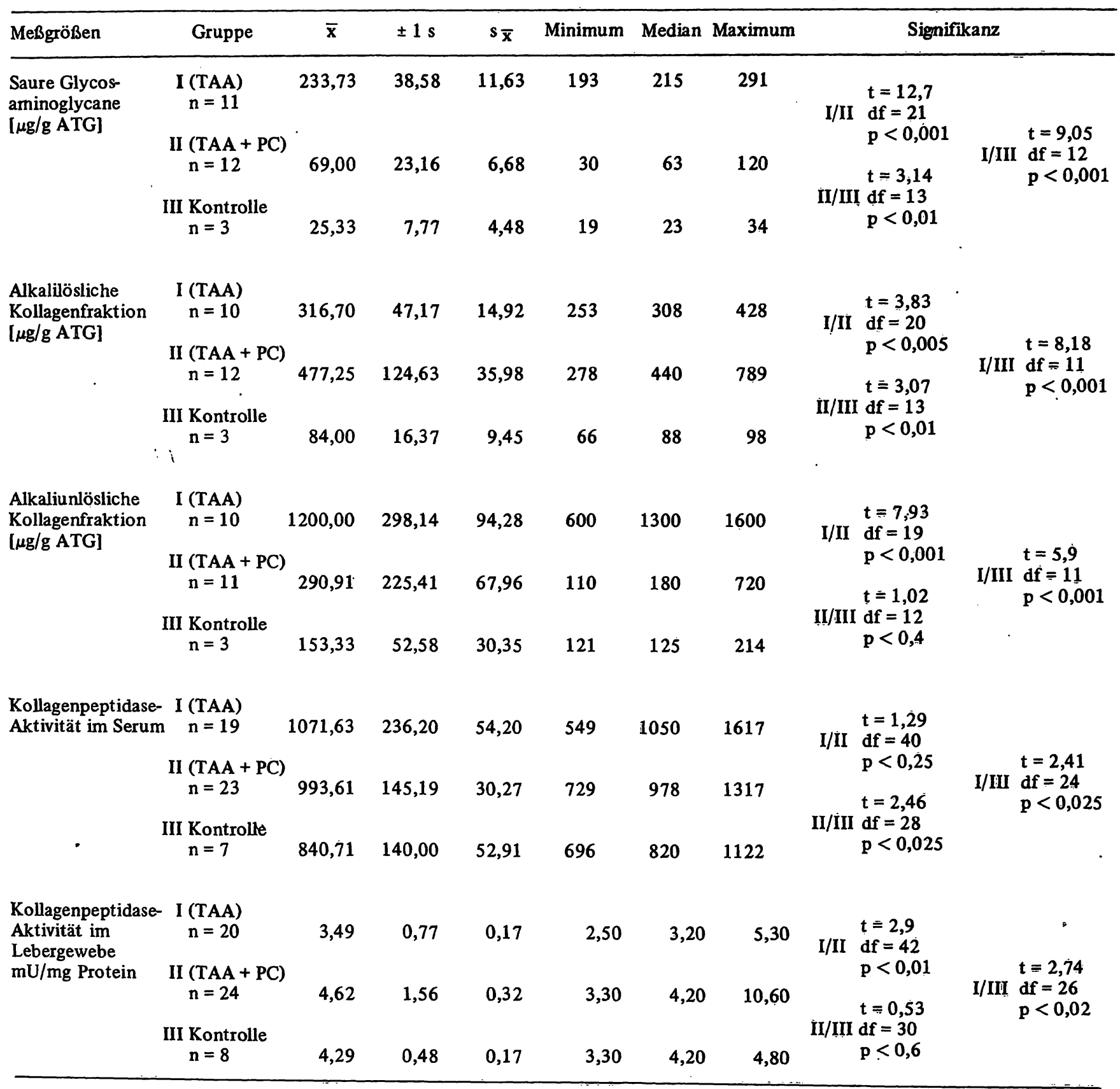

Gruppe I betrug die Konzentration der sauren Glykosaminoglykane im Mittel fast das 10-fache derjenigen der weder mit Thioacetamid noch mit Pyridinolcarbamat behandelten Kontrollgruppe (III). In der mit Pyridinolcarbamat behandelten Versuchsgruppe (II) lagen die Werte der sauren Glykosaminoglykanspiegel in den Lebern um das Dreifache unter denjenigen der Gruppe I. Die elektrophoretische Auftrennung der Glykosaminoglykanfraktionen zeigte in allen Untersuchungsgruppen ein gleiches qualitatives und quantitatives Verteilungsmuster.

Ebenfalls signifikant unterschieden sich die Gewebsspiegel der alkaliunlöslichen Kollagenfraktion, gemessen am Hydroxyprolinspiegel in den Gruppen I, II sowie im Vergleich zur Kontrollgruppe III, jedoch ergàb sich entsprechend der angewandten Methode ein etwas größerer Streubereich. Im Gegensatz dazu ließ sich ein signifikanter Unterschied in den Gewebsspiegeln der alkalilöslichen 

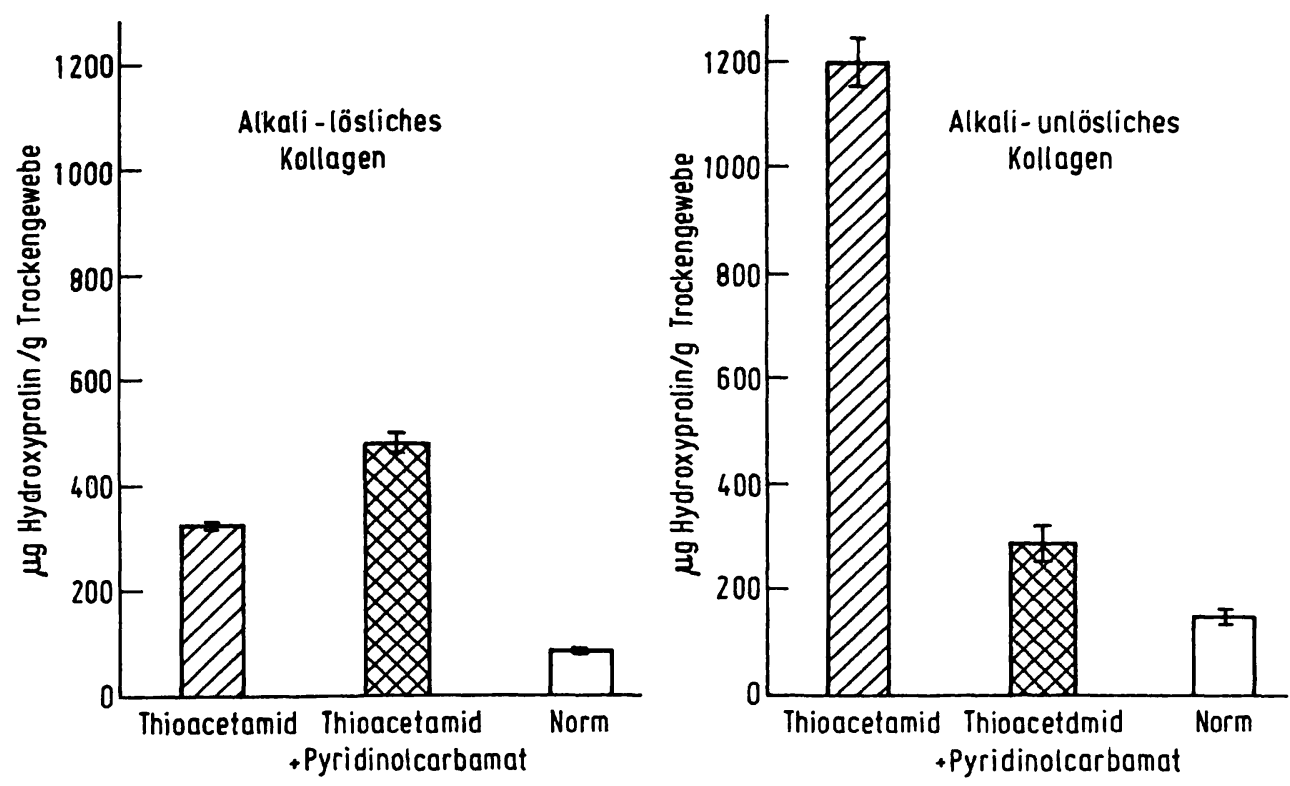

Abb. 3. Gewebsspiegel der Kollagenfraktionen: $0,5 \mathrm{~mol} / 1 \mathrm{NaOH}$ löslich und 0,5 mol/1 $\mathrm{NaOH}$ unlöslich I = Standardabweichung der Mittelwerte
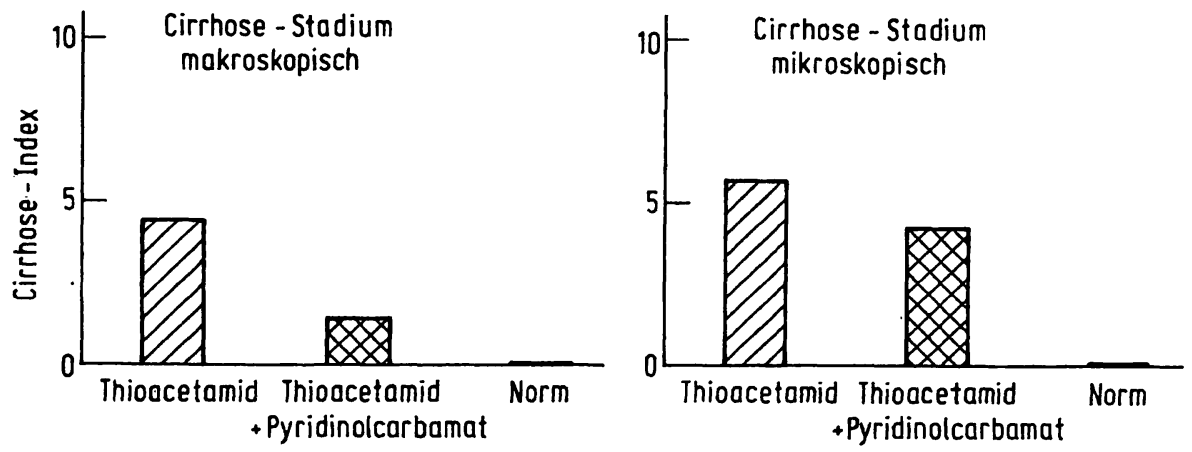

Abb. 4. Durchschnittliche mikroskopische und makroskopische Bewertung der Leberpräparate der Versuchsgruppen. Cirrhoseindex: Mittelwert der morphologischen Bewertungsziffer (s. Text).

Fraktion zwischen den Gruppen I und II nicht erkennen (Tab. 2, Abb. 3). Die Aktivität der Kollagenpeptidase, die bei proliferativen Bindegewebsprozessen in Geweben und im Blutserum erhöht gefunden wird $(18,19,20)$, zeigte keine Unterschiede in den Lebergewebsproben und den Blutseren der Tiere der 3 Gruppen (Tab. 2). Zur biochemischen Beurteilung des Leberparenchyms wurden die Enzymaktivitäten von Glutamatoxalacetattransami; nase, Glutamatpyruvattransaminase, Glutamatdehydrogenase und der alkalischen Phosphatase im Gewebe und im Blutserum bestimmt.sowie der Bilirubingehalt des Blutserums ermittelt. Die Ergebnisse sind in Tabelle 3 dargelegt. Die Aktivitätsmuster entsprachen mit einer geringen Erhöhung der Glutamatoxalacetattransaminase und einem Anstieg der Glutamatdehydrogenase und alkalischen Phosphatase-Aktivitäten im Serum den Verhältnissen beim Menschen im Verlaufe einer Lebercirrhose. Der Bilirubinspiegel im Serum war in den Gruppen I und
II auf etwa das Vierfache der Kontrollgruppe erhöht. Unterschiede zwischen den Gruppen I und II fanden sich nicht. Im Lebergewebe der beiden Gruppen I und II lagen die entsprechenden Enzymaktivitäten gegenüber der Kontrollgruppe mäßig erniedrigt, wobei allerdings die Glutamatoxalacetattransaminase in der Gruppe II nicht von der Glutamatoxalacetattransaminaseaktivität der Kontrollgruppe, aber von der der Vergleichsgruppe I unterschieden war: Die an den gleichen Gruppen bestimmten Cholesterinspiegel im Serum zeigten keine Beeinflussung für die verabfolgten Substanzen, so daß wir das auch von anderen Autoren $(2,3)$ berichtete Fehlen einer Beeinflußbarkeit des Cholesterinserumspiegels durch Pyridinolcarbamat bestätigen konnten.

Die zur Kontrolle der Aufnahme von Thioacetamid und Pyridinolcarbamat durchgeführte dünnschichtchromatographische Untersuchung von Leberextrakten schloß eine gegenseitige Beeinflussung beider Substanzen aus 
und ließ auf annähernd gleiche Gewebsspiegel furr Thioacetamid in der Versuchs- und Vergleichsgruppe schliessen.

\section{Besprechung der Ergebnisse}

Die unkontrollierte Proliferation des Bindegewebes bei der durch einen primären Parenchymzellschaden verursachten Lebercirrhose fuihrt zur Ausbildung eines zellarmen Narbengewebes in der betroffenen Leber. Neben der morphologischen Charakterisierung des Prozesses bieten sich die Gewebsspiegel der interzellulären Struktursubstanzen der Bindegewebe als biochemische Parameter der Bindegewebsbildung an. Die quantitative Bestimmung der sauren Glykosaminoglykane und des $\mathrm{Hy}$ dryxyprolins als Maß für den Anteil der Faserproteine geben einen guten Einblick in die Entwicklung der Lebercirrhose. Trotz methodischer Bedenken kann auf die relativ wenig aufwendige Differenzierung der Kollagenfraktionen aufgrund ihrer unterschiedlichen Löslichkeiten in Neutralsalzlösungen bzw. saurem oder alkalischem Milieu in der Reihenuntersuchung nicht verzichtet werden. Dabei ist das gewählte Extraktionsverfahren maßgebend für die Interpretation derartiger Untersuchungsergebnisse. Das hier zur Untersuchung kommende Lebermaterial erfordert die Anwendung der Kollagenextraktion mit $0,5 \mathrm{~mol} / 1 \mathrm{NaOH}$, da der hohe Nichtkollageneiweißanteil der Leber bei weniger stark wirksamen Extraktionsmedien keine verwertbaren Resultate erwarten läßt (18). Die Verteilung der löslichen und unlöslichen Hydroxypyrolin-Proteine entspricht bei diesem Vorgehen derjenigen, die mit $8 \mathrm{~mol} / 1 \mathrm{Harnstoff}$ bei $40^{\circ} \mathrm{C}$ erzielt wird $(19,20)$. Diese methodischen Gegebenheiten erklären den relativ hohen Anteil „löslicher Kollagenproteine" am Gesamtkollagengehalt der Untersuchungsproben. Bei der Interpretation der Ergebnisse muß man sich vor Augen halten, daß neben Prokollagen in nicht unerheblichem Maße Kollagen in der mit $0,5 \mathrm{~mol} / 1 \mathrm{NaOH}$ extrahierten Fraktion enthalten ist. Diese Kollagenanteile sind entweder noch nicht fest an die Faser gebunden oder ihre Bindungsfähigkeit ist pathologisch gestört. Die sauren Glykosaminoglykane liegen in der Grundsubstanz der Bindegewebe als Proteinkomplex, zum Teil in Hybridform, vor und bestimmen aufgrund ihrer spezifischen Funktion wesentlich die physikalisch-chemischen Eigenschaften dieser Grundsubstanz. Der quantitativen Erfassung dieser Proteoglykan-Komplexe stellen sich erhebliche methodische Schwierigkeiten, besonders im Hinblick auf den Gewebsaufschluß des nur in kleinsten Mengen Glykosaminoglykane enthaltenden Lebergewebes entgegen. Daher ist der Isolierung der sauren Glykosaminoglykane nach proteolytischem Gewebsaufschluß der Vorzug zu geben. Gegenüber dem in einer früheren Mitteilung (8) angegebenen Verfahren zur Isolierung und quantitativen Erfassung der sauren Glykosaminoglykane konnte bei diesen Untersuchungen durch die Einbeziehung eines Gelfiltrationsschrittes in den Isolierungsgang die Zuverlässigkeit der Methode gegenüber den klassischen Fällungsverfahren wesentlich verbessert werden, da durch sie alle unspezifischen Chromogene einschließlich der Nicht-Glykosaminoglykan-Uronide abgetrennt werden können (Abb. 5).

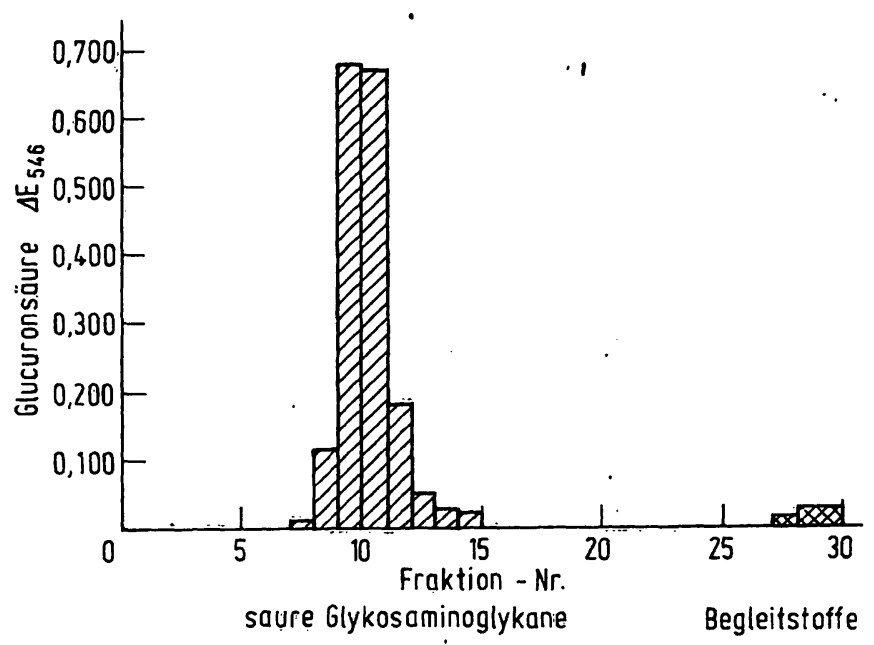

Abb. 5. Gelfiltration der Glykosaminoglykan-Rohfraktion an Sephadex G-25-fein, Elution mit demineralisierten Wasser. Abtrennen von Begleitstoffen, insbesondere Nicht-Glykosaminoglykan-Glucuroniden.

Auf eine Aktivierung des Stoffwechsels der sauren Glykosaminoglykane im Rahmen spezifischer und unspezifischer Mesenchymreaktionen (21) weisen die gesteigerten Einbauraten von ${ }^{35} \mathrm{~S}$-Sulfatschwefel in die sauren Glykosaminoglykane betroffener Gewebe (22) sowie erhöhte Ausscheidungsraten der sauren Glykosaminoglykane im Harn (23-25) hin. Bei quantitativer Bestimmung der sauren Glykosaminoglykane nach Isolierung aus cirrhotischen Lebern von Menschen und Ratten fanden Becker (26) wie Delbrück (8) die Konzentration gegenüber der normalen Vergleichsprobe um ein Mehrfaches erhöht. Eine Depression sowohl der Einbauraten von ${ }^{35}$ S-Sulfat (27) wie der Konzentration der sauren Glykosaminoglykane bei der Thioacetamidlebercirrhose der Ratte konnte durch Proliferationshemmer bzw. antiinflammatorische Wirkstoffe (Corticoide, Phenylbutazion) nachgewiesen werden $(6,26,27)$. Wie von Rave et al (28) und in dieser Untersuchung gezeigt, übt Pyridinolcarbamat eine gleichsinnige Wirkung auf proliferative Bindegewebsprozesse aus. Pyridinolcarbamat verhindert nicht die Leberparenchymschädigung wie aus den in Tabelle 3 aufgezeichneten Daten hervorgeht. Auch eine Mesenchymreaktion ist mikroskopisch nachweisbar. Offensichtlich kommt es unter Pyridinolcarbamat zu einer Supprimierung der Proliferation und insbesondere der Synthese und Ablagerung von Grundsubstanz (saure Glykosaminoglykane) sowie Faserproteinen (Kollagen). Dabei wird eine Veränderung im Verteilungsmuster der sauren Glykosaminoglykanfraktionen im Vergleich zur Thioacetamidgruppe (I) wie żu der Kontrollgruppe (III) nicht beobachtet. Auch in früheren Untersuchungen $(6,8)$ wurden lediglich quantitative, nicht qualitative 
Tab. 3. Biochemische Parameter zur Beurteilung des Leberparenchyms.

\begin{tabular}{|c|c|c|c|c|c|c|}
\hline Gruppe & $\begin{array}{c}\text { Serum } \\
\text { U/1 } \\
\quad I\end{array}$ & $\begin{array}{l}\text { Leber-Extrakt } \\
\text { U/mg Protein } \\
\text { I }\end{array}$ & $\begin{array}{l}\text { Serum } \\
\text { U/1 } \\
\text { II }\end{array}$ & $\begin{array}{c}\text { Leber-Extrakt } \\
\text { U/mg Protein } \\
\text { II }\end{array}$ & $\begin{array}{l}\text { Serum } \\
\text { U/1 } \\
\text { III }\end{array}$ & $\begin{array}{c}\text { Leber-Extrakt } \\
\text { U/mg Protein } \\
\text { III }\end{array}$ \\
\hline $\begin{array}{l}\text { Glutamat-Oxalacetat- } \\
\text { Transaminase }\end{array}$ & $\begin{array}{r}138 \pm 36 \\
n=21\end{array}$ & $\begin{aligned} 882 & \pm 212 \\
n & =19\end{aligned}$ & $\begin{array}{r}115 \pm 19 \\
n=24\end{array}$ & $\begin{aligned} 1236 & \pm 301 \\
n & =24\end{aligned}$ & $\begin{aligned} 105 & \pm 21 \\
n & =6\end{aligned}$ & $\begin{aligned} 1375 & \pm 218 \\
n & =7\end{aligned}$ \\
\hline $\begin{array}{l}\text { Glutamat-Pyruvat- } \\
\text { Transaminase }\end{array}$ & $\begin{aligned} 17 & \pm 5,1 \\
\mathrm{n} & =20\end{aligned}$ & $\begin{array}{r}101 \pm 26 \\
n=20\end{array}$ & $\begin{array}{c}14 \pm 3,5 \\
n=23\end{array}$ & $\begin{array}{r}97 \pm 25 \\
n=24\end{array}$ & $\begin{aligned} 16 & \pm 3,1 \\
n & =4\end{aligned}$ & $\begin{aligned} 210 & \pm 65 \\
n & =5\end{aligned}$ \\
\hline Glutamat-Dehydrogenase & $\begin{aligned} 23 & \pm 7,5 \\
n & =13\end{aligned}$ & $\begin{array}{c}1,47 \pm 0,6 \\
n=19\end{array}$ & $\begin{aligned} 26 & \pm 13,8 \\
n & =25\end{aligned}$ & $\begin{aligned} 1,17 & \pm 0,5 \\
n & =24\end{aligned}$ & $\begin{aligned} 8,8 & \pm 1,6 \\
n & =5\end{aligned}$ & $\begin{array}{c}1,48 \pm 0,6 \\
n=5\end{array}$ \\
\hline alkalische Phosphatase & $\begin{array}{r}258 \pm 70 \\
n=19\end{array}$ & - & $\begin{array}{r}212 \pm 49 \\
n=25\end{array}$ & - & $\begin{aligned} 164 & \pm 24,5 \\
\mathrm{n} & =6\end{aligned}$ & - \\
\hline Bilirubin mmol/1 & $\begin{aligned} 17,1 & \pm 4,5 \\
n & =21\end{aligned}$ & - & $\begin{array}{c}16,5 \pm 4,6 \\
n=25\end{array}$ & - & $\begin{aligned} 4,41 & \pm 1,15 \\
n & =6\end{aligned}$ & \\
\hline
\end{tabular}

Veränderungen im Gehalt an sauren Glykosaminoglykanen bei der Entwicklung einer Lebercirrhose bei Mensch und Ratte gefunden. Diese Ergebnisse stehen im Gegensatz zu der Mitteilung von Becker (26), der seine Befunde an menschlichen Lebern dahingehend deutet, daß die Vermehrung der sauren Glykosaminoglykane zum wesentlichen Teil auf der Zunahme des Dermatansulfates beruhen. Diese Diskrepanz kann methodisch gedeutet werden, da in einem Fall ein indirektes Verfahren (26) im anderen Falle die Isolierung der einzelnen Glykosaminoglykanfraktionen vorgenommen wurde (8). Die mit der cirrhotischen Umwandlung des Lebergewebes verbundenen strukturellen Veränderungen lassen neben einer Steigerung von Synthese und intrazellulärer Ablagerung eine wenn auch geringe Erhöhung der Kollagenabbauraten erwarten. Für eine Reihe proliferativer Bindegewebsprozesse wurd aufgrund erhöhter Ausscheidungsraten von Kollagenpeptiden im Harn und gesteigerter Kollagenpeptidaseaktivität auf das gleichzeitige Vorliegen eines gesteigerten Kollagenabbaus geschlossen. So wird u. a. von Müller et al $(27,30)$ über eine erhöhte Kollagenpeptidaseaktivität im Serum bei der Sarcoidose wie in der Synovialmembran bei chronischer Arthritis (31) berichtet. Im Gegensatz zu diesen Befunden konnten wir an der Ratte bei der experimentellen Lebercirrhose keine Unterschiede in der Aktivität dieses Enzyms im Leberge- webe wie im Blutserum der drei untersuchten Gruppen finden.

Pyridinolcarbamat hat sich aufgrund der vorliegenden Untersuchungen am Modell der Bindegewebsbildung bei der durch Thioacetamid ausgelösten Lebercirrhose der Ratte als Inhibitor der Bildung und Deponierung von Faserproteinen und Grundsubstanzanteilen erwiesen. Aufgrund dieses Ergebnisses kann Pyridinolcarbamat den Antiproliferativa zugeordnet werden.

Weiterhin stützen die hier mitgeteilten Ergebnisse die eingangs angeführte Hypothese einer antiatherogenen Wirksamkeit von Pyridinolcarbamat durch Einflußnahme auf den Stoffwechsel des Gefäßbindegewebes. Eine differente Wirkung des Pyridinolcarbamates auf die Synthese der Glykosaminoglykane einerseits und der Faserproteine andererseits läßt sich nicht ableiten. Eine weitere Differenzierung der biochemischen Wirkungsweise von Pyridinolcarbamat ist zusätzlichen Untersuchungen vorbehalten.

\section{Danksagung}

Der Autor ist Herrn Dr. Feldmann, Department für Biometrie und medizinische Informatik der Med. Hochschule Hannover für die mathematisch-statistische Beratung und Bearbeitung der Versuchsdaten zu großem Dank verpflichtet.

Frl. Marianne Arlart und Herrn Heinrich Schröder gilt der besondere Dank für sorgfältige technisch-experimentelle Mitarbeit bei der Versuchsdurchführung.

\section{Literatur}

1. Shimamoto, T., Numano, F., Fujita, T., Ishioka, T. \& Atsumin, T. (1965) Asian Med. J. 8, 12, (Zitat nach 2)

2. Shimamoto, T. \& Numano, R. (Hrsg.) (1969) Atherogenesis I, Excerpta Medica Foundation, Amsterdam

3. Shimamoto, T., Numano, F. \& Addison, G. M. (Hrsg.) (1973) Atherogenesis II, Excerpta Medica Foundation, Amsterdam

4. Yamamura, T., Inhibitor effects of pyridinolcarbamate in angiolathyrism and osteolathyrism in 1.c. 2, S. 29-36

5. Brodehl, I. (1961) Klin. Wochenschr. 39, 956-962
6. Arnold, H., Delbrück, A. \& Hartmann, F. (1963) Deut. Arch. Klin. Med. 209, 92-116

7. Stegemann, H. (1958) Hoppe-Seyler's Z. Physiol. Chem., $311,41-45$

8. Delbrück, A. (1968) diesc Z. 6, 460-466

9. Dische, Z. (1947) J. Biol. Chem. 167, 189-198

10. Schmidt, E. \& Schmidt, F. W. (1962/63) Enzym. Biol. Clin. $2,201-222$ 
11. Hausamen, T. V., Helger, R., Rick, W. \& Gross, W. (1967) Clin. Chim. Acta 15, 241-245

12. Wünsch, E. \& Heidrich, H. G. (1963) Hoppe-Seyler's Z. Physiol. Chem. 332, 300-304

13. Wünsch, E. \& Heidrich, H. G. (1963) Hoppe-Seyler's Z. Physiol. Chem. 333, 149-151

14. Strauch, L. \& Vencelj, H. (1967) Hoppe-Seyler's Z. Physiol. Chem. 348, 465-468

15. Persönliche Mitteilung Kali-Chemie AG, Hannover, Wiss. Abteilung

16. Jendrassik, L \& Grof, P. (1968) Biochem. Z. 297, 81-89

17. Watson, D. (1960) Clin. Chim. Acta 5, 637-643

18. Hirayama, C. \& Hartmann, F. (1961) Acta Hepatosplenol. (Stuttgart) 8, 102-109

19. Zimmermann, B. K. \& Balda, B. R. (1972) Arch. Derm. Forsch. 243, 357-363

20. Veis, A. \& Asney, L. (1965) J. biol. Chem. 240, 3899-3908

21. Hauss, W. H., Junge-Hülsing, G. \& Gerlach, U. (1968) Die unspezifische Mesenchymreaktion, Thieme, Stuttgart
22. Junge-Hülsing, G. (1960), in Struktur und Stoffwechsel des Bindegewebes (Hauss, W. H. \& Losse, H., Hrsg.) S. 83-97. Thieme, Stuttgart

23. Delbrück, A., Grumme., H. \& Wette, K. (1967) diese Z. 5, 16-21

24. Murata, K., Ishikawa, T. \& Oshima, Y. (1970) Clin. Chim. Acta 28, 213-222

25. Bitter, T., Siegenthaler, P., de Preux, T. \& Martin, E. (1970) Ann. Rheum. Dis. 29, 427-433

26. Becker, K. (1967) Z. Gesamte Exp. Méd. 144, 222-235

27. Becker, K. (1967) Verh. Deut. Ges. Inn. Med. 73, 264-267

28. Rave, O., Wagner, H., Hrubesch, M., Junge-Hülsing, G. \& Hauss, W. H., Arzneimittelforschung, im Druck

29. Stojan, B., Müller, W., Wurm, K. \& Tariverdian, M. (1973) Schweiz. Med. Wochenschr. 103, 337-341

30. Müller, W., Wurm, K., Stojan, B. \& Tariverdian, M. (1973) Deut. Med. Wochenschr. 98, 1253-1255

31. Stojan, B., Müller, W. \& Verasani, D. (1971) in Rheumatoid Arthritis (Müller, W., Harwerth, H. G. \& Fehr, K. (Hrsg.) S. 169-171 Academic Press, Lọndon/New York

Prof. Dr. A. Delbrück 3000 Hannover Podbielskistr. 380 